\title{
Evaluation of Impact of Interferon-Induced Helicase C Domain-Containing Protein 1 Gene in Egyptian Systemic Lupus Erythematosus Patients and its Relationship With Vascular Manifestations of the Disease
}

\author{
Sahar NASSEF, ${ }^{1}$ Mervat ESSAM, ${ }^{2}$ Dina ABDELFATTAH, ${ }^{3}$ Doaa ELSEHEMY, ${ }^{2}$ Sahar ABOUELEZZ ${ }^{2}$ \\ ${ }^{1}$ Department of Internal Medicine, Faculty of Medicine Cairo University, Vascular Division, Cairo, Egypt \\ ${ }^{2}$ Department of Internal Medicine, Division of Rheumatology \& Clinical Immunology, Faculty of Medicine Cairo University, Cairo, Egypt \\ ${ }^{3}$ Department of Medical Biochemistry and Molecular Biology, Faculty of Medicine Cairo University, Cairo, Egypt
}

\begin{abstract}
Objectives: This study aims to investigate the impact of interferon-induced helicase C domain-containing protein 1 (IFIH1) gene single nucleotide polymorphism (SNP) on interferon pathway signaling in systemic lupus erythematosus (SLE) patients specifically with vascular affection.

Patients and methods: The cross-sectional study included 30 consecutive SLE patients ( 2 males, 28 females; mean age 28 \pm 3.4 years; range, 16 to 40 years) diagnosed according to the American College of Rheumatology revised criteria and 10 healthy age- and sex-matched controls ( 2 males, 8 females; mean age $27 \pm 2.5$ years; range 22 to 23 years). SLE patients and controls were compared in terms of quantitative reverse transcriptase polymerase chain reaction gene expression of IFIH1 gene, von Willebrand factor, carotid intima-media thickness, and Ankle Brachial Index.

Results: Interferon-induced helicase C domain-containing protein 1 gene expression was significantly higher in SLE patients than controls (1.7 \pm 0.6 and $0.5 \pm 0.2$, respectively) $(p<0.0001)$. IFIH1 gene expression was highly related to vascular complication with a cutoff point at 1.74 and it positively correlated with other endothelial dysfunction markers.

Conclusion: Interferon-induced helicase C domain-containing protein 1 gene (SNP 1990670) is associated with SLE in Egyptian patients. Expression of IFIH1 gene is related to disease activity and may serve as a novel predictor of vascular affection in SLE.

Keywords: Interferon-induced helicase $\mathrm{C}$ domain-containing protein 1 gene; vascular; von Willebrand factor.
\end{abstract}

Systemic lupus erythematosus (SLE) is a common worldwide inflammatory autoimmune disorder. The pathogenesis of SLE remains not fully understood; it is known to depend upon the interaction of genetic, environmental and hormonal factors. ${ }^{1}$ However, to date, the most valid evidence indicates that it is mainly due to the deficiency of several immunological mechanisms, including inappropriate function of the innate immune system, altered self-tolerance mechanisms and apoptotic cell clearance. ${ }^{2}$

Accelerated subclinical atherosclerosis and vascular complications in patients with SLE seem to be attributed to other risk factors rather than the traditional Framingham risk factors such as apoptosis and endothelial dysfunction. ${ }^{3}$ Endothelial dysfunction may result from disrupted endothelial progenitor cell activity, which is mediated by type 1 interferon (IFN) that has proinflammatory and antiangiogenic effects in SLE. ${ }^{4}$

Interferon-induced helicase $\mathrm{C}$ domaincontaining protein 1 (IFIH1), which is known as melanoma differentiation-associated gene 5 or Helicard, is located at 2q24.3 and encodes type $1 \mathrm{IFN}$ and IFN-induced gene. This gene was implicated in other autoimmune diseases such as 
autoimmune thyroiditis and psoriasis, while being considered as a gene proposed to improve the prediction of type 1 diabetes mellitus (DM). ${ }^{5}$

In a study, Wang et al. ${ }^{6}$ reported the contribution of IFIH1 in systemic lupus susceptibility. Many studies also suggested that IFIH1 gene has a role in inflammation and apoptosis in SLE, ${ }^{7}$ so IFIH1 might be related to endothelial dysfunction, increased plasma levels of von Willebrand factor (vWF) and in return, atherosclerosis in SLE. ${ }^{8}$

Intima-media thickness (IMT) is a surrogate marker for atherosclerosis and was shown to be elevated in lupus patients compared to age- and sex-matched controls. ${ }^{9}$ Thus, it may serve as a solid gold standard for detecting atherosclerosis in SLE patients. Therefore, in this study, we aimed to investigate the impact of IFIH1 gene single nucleotide polymorphism (SNP) on interferon pathway signaling in SLE patients specifically with vascular affection.

\section{PATIENTS AND METHODS}

This cross-sectional study was conducted at Kasr Al-Ainy Hospital between August 2015 and September 2016 and included 30 SLE patients (2 males, 28 females; mean age $28 \pm 3.4$ years; range 16 to 40 years) fulfilling the American College of Rheumatology criteria excluding any patients with other autoimmune diseases, type $1 \mathrm{DM}$, thyroid diseases or malignancy. Patients were selected from the outpatient Rheumatology and Immunology Clinic and Internal Medicine Department of Kasr Al-Ainy Hospital and compared to 10 healthy age- and sex-matched control subjects ( 2 males, 8 females; mean age $27 \pm 2.5$ years; range, 22 to 23 years). The study protocol was approved by the Cairo University Ethics Committee. A written informed consent was obtained from each patient. The study was conducted in accordance with the principles of the Declaration of Helsinki.

All subjects enrolled in the study underwent full history taking and complete physical examination, with special emphasis on symptoms of lupus activity which was recorded for each patient using the Systemic Lupus Erythematosus Disease Activity Index (SLEDAI) score.

A total of $20 \mathrm{~mL}$ blood was withdrawn from all subjects. Complete blood count was estimated using a cell counter by CELL-DYN machine (Abbott, Illinois, USA), erythrocyte sedimentation rate was estimated by Westergren's method, C-reactive protein by neplometery BN II nephelometer (Siemens, Germany), anti-nuclear autoantibodies by indirect immunofluorescence, anti-double stranded deoxyribonucleic acid (DNA) by immunofluorescence, and $\mathrm{C} 3-\mathrm{C} 4$ by radial immunodiffusion.

Anticardiolipin was evaluated by enzymelinked immunosorbent assay (ELISA) and lupus anticoagulant testing. Serum creatinine was estimated by kinetic method over automation system by Dimension machine (Siemens Healthcare $\mathrm{GmbH}$, Germany), urine analysis, and 24-hour urinary protein. Serum cholesterol, triglyceride, low density lipoprotein (LDL) and high density lipoprotein portions of cholesterol were also estimated.

Interferon-induced helicase $\mathrm{C}$ domaincontaining protein 1 gene quantitative assessment was performed by real-time polymerase chain reaction (RT-PCR) as follows: peripheral blood was obtained from SLE patients and healthy controls. Peripheral blood mononuclear cells were isolated by a Ficoll-Paque density gradient (Pharmacia Biotech, Uppsala, Sweden) to remove erythrocytes, platelets, and cell debris. Total ribonucleic acid (RNA) was extracted using QIAGEN RNeasy mini kit (Qiagen GmbH,

Table 1. Primers sequence and annealing temperature specific for each gene

\begin{tabular}{lll}
\hline Target gene & Primer sequence: 5` ${ }^{`}$ & Gene bank accession number \\
\hline IFIH1* & $\begin{array}{l}\text { Forward: ATGGAAAAAAAAGCTGCAAAAGA } \\
\text { Reverse: GTACTTCCTCAAATGTTCTGCACAA }\end{array}$ & NM_022168.3 \\
GAPDH & $\begin{array}{l}\text { Forward: CCTCTACTGGCGCTGCCAAGGCT } \\
\text { Reverse: GTCCACCACTGACACGTTGG }\end{array}$ & NT_009759.16 \\
\hline "IFIH: Interferon-induced helicase C domain-containing protein 1; GAPDH: Glyceraldehyde-3-phosphate dehydrogenase.
\end{tabular}


Table 2. Distribution of systemic lupus erythematosus patients as regards to clinical presentation

\begin{tabular}{lcc}
\hline Clinical manifestations & $\mathrm{n}$ & $\%$ \\
\hline Musculoskeletal & 28 & 93.3 \\
Alopecia & 17 & 56.7 \\
Malar rash \& photosensitivity & 21 & 70 \\
Oral ulcer & 18 & 60 \\
Serositis & 4 & 13.3 \\
Neuropsychiatric (other than vascular) & 3 & 10 \\
Vascular & & \\
$\quad$ No vascular affection & 20 & 66.7 \\
Venous & 6 & 20 \\
$\quad$ Arterial & 4 & 13.3 \\
Nephritis & 19 & 63.3 \\
Raynaud's phenomenon & 9 & 30 \\
\hline
\end{tabular}

Hilden, Germany). Reverse transcription was performed on deoxyribonuclease-treated total RNA. Complementary DNA was synthesized from messenger RNA with oligo(dT) primer and Superscript II Reverse Transcriptase (Invitrogen, Grand Island, NY, USA). Specific primers and probes were used in the RT-PCR assay (Table 1). RT-PCR was performed by One-Step Applied Biosystems RT-PCR (Foster City, CA, USA). The relative changes of IFIH1 human gene were analyzed by SYBR Green RT-PCR (StepOne ${ }^{\mathrm{TM}}$, USA). Dissociation curve analysis was performed after each assay to ensure specific target detection.

After the RT-PCR, the data were expressed in cycle threshold (Ct). The PCR data sheet includes $\mathrm{Ct}$ values of the assessed IFIH1 gene and the house-keeping (reference) gene -the gene expressed in the cell continuously and normally. In order to measure the gene expression of a certain gene, a negative sample as control should be used. Thereafter, target gene expression was assessed and related to reference (internal control) gene. Finally, the respiratory quotient (RQ) was calculated according to the following equation:

1. $\Delta \mathrm{Ct}=\mathrm{Ct}_{\text {assessed gene }}-\mathrm{Ct}_{\text {reference gene }}$

2. $\Delta \Delta \mathrm{Ct}=\Delta \mathrm{Ct}_{\text {sample }}-\mathrm{Ct}_{\text {control gene }}$

3. $\mathrm{RQ}=2-(\Delta \Delta \mathrm{Ct})$

von Willebrand factor assessment was performed by ELISA technique using Technoclone $\mathrm{GmbH}$ (Vienna, Austria). Carotid IMT and Ankle Brachial Index (ABI) measurements were conducted by using an ultrasound machine (Siemens Acuson Antares; Siemens Healthcare $\mathrm{GmbH}$, Erlangen. Germany). The transducer used to assess the carotid Doppler was 7.5 MHZ. We assessed the carotid IMT on the selected subjects in supine position with the head slightly extended and turned to the opposite direction of the common carotid artery (CCA) being studied. Both sides were imaged. On a longitudinal, twodimensional ultrasound image of CCA, each anterior (near) and posterior (far) wall of the CCA was displayed as two bright white lines separated by a hypoechoic space. The distance between the leading edge of the first bright line (the bloodintima interface) of the far wall and the leading edge of the second bright line (media-adventitia interface) indicates the IMT.

Ankle Brachial Index was measured while the subjects were at rest in supine position for 10 minutes. $\mathrm{ABI}$ is equal to the systolic pressure at the ankle, divided by the systolic pressure at the arm (systolic pressures were recorded with a handheld 5- or $10 \mathrm{mHz}$ Doppler instrument).

Table 3. Comparison between systemic lupus erythematosus patients and controls as regards to laboratory and vascular laboratory data

\begin{tabular}{lcc}
\hline Variables & SLE patients $(\mathrm{n}=30)$ & Controls $(\mathrm{n}=10)$ \\
\cline { 2 - 3 } & Mean $\pm \mathrm{SD}$ & Mean $\pm \mathrm{SD}$ \\
\hline Fasting glucose $(\mathrm{mg} / \mathrm{dL})$ & $92 \pm 10$ & $90 \pm 11$ \\
Total cholesterol (mg/dL) & $197 \pm 20$ & $120 \pm 26$ \\
HDL (mg/dL) & $53.9 \pm 23$ & $36 \pm 10$ \\
LDL (mg/dL) & $100 \pm 48$ & $61 \pm 30$ \\
Triglyceride (mg/dL) & $148 \pm 50$ & $102 \pm 44$ \\
IFIH1 gene & $1.7 \pm 0.6$ & $0.49 \pm 0.21$ \\
vWF (IU/mL) & $5.9 \pm 1.8$ & $1.8 \pm 0.8$ \\
Average carotid intima media thickness (mm) & $6.2 \pm 2$ & $4.6 \pm 1.5$ \\
ABI & $0.9 \pm 0.1$ & $1.1 \pm 0.5$ \\
\hline SLE: Systemic lupus erythematosus; SD: Standard deviation; HDL: High density lipoprotein; LDL: Low density lipoprotein; \\
IFIH1: Interferon-induced helicase C domain-containing protein 1; vWF: von Willebrand factor; ABI: Ankle Brachial Index.
\end{tabular}


Table 4. Correlation between interferon-induced helicase $C$ domain-containing protein 1 , von Willebrand factor and different variables among systemic lupus erythematosus patients

\begin{tabular}{lcccc}
\hline Variables & \multicolumn{2}{c}{ IFIH1 } & & vWF \\
\cline { 2 - 5 } & r-value & $p$ & r-value & $p$ \\
\hline Age (year) & 0.12 & 0.56 & 0.10 & 0.50 \\
Disease duration & 0.10 & 0.60 & 0.11 & 0.34 \\
Systemic Lupus Erythematosus Disease Activity Index & $\mathbf{0 . 5 4}$ & $\mathbf{0 . 0 0 2}$ & -0.04 & 0.62 \\
Cholesterol (mg/dL) & 0.03 & 0.78 & 0.06 & 0.75 \\
High density lipoprotein (mg/dL) & 0.11 & 0.64 & 0.12 & 0.60 \\
Low density lipoprotein (mg/dL) & 0.10 & 0.50 & 0.15 & 0.20 \\
Triglyceride (mg/dL) & 0.18 & 0.35 & 0.19 & 0.45 \\
Ankle Brachial Index & $\mathbf{- 0 . 3 4}$ & $\mathbf{0 . 0 5}$ & $\mathbf{0 . 5 5}$ & $\mathbf{0 . 0 0 2}^{*}$ \\
Carotid intima-media thickness (mm) & 0.22 & 0.25 & 0.20 & 0.33 \\
von Willebrand factor (IU/mL) & $\mathbf{0 . 5 7}$ & $\mathbf{0 . 0 0 1}^{*}$ & & \\
\hline IFIH1: Interferon-induced helicase C domain-containing protein 1; vWF: Von Willebrand factor; * Significant. & & \\
\hline
\end{tabular}

Ankle brachial pressure index for each leg was calculated using the equation:

$$
\mathrm{ABPI}_{\text {Leg }}=\frac{\mathrm{P}_{\text {Leg }}}{\mathrm{P}_{\text {Arm }}}
$$

$\mathrm{P}_{\text {Leg }}$ is the systolic blood pressure of dorsalis pedis or posterior tibial arteries and $P_{A r m}$ is the highest of the left and right arm brachial systolic blood pressure.

\section{Statistical analysis}

Quantitative variables were expressed as mean, standard deviation and range. Qualitative variables were described as number and percentage. Chi-square test was used to compare qualitative variables between groups. Unpaired t-test was used to compare two groups as regards to quantitative variables. Correlation coefficient test was used to identify the negative or positive corrletion between different variables. Receiver operator characteristic curve was used to find out the best cutoff value, and validity of a certain variable. $\mathrm{P}$ value was considered significant if $<0.05$, highly significant if $<0.01$, and very highly significant if $<0.001$.

\section{RESULTS}

Disease duration of the patients was mean $43 \pm 40$ months (range 3 month to 9 years). Systemic lupus disease activity index score ranged between 2-16 with a mean of $7 \pm 3.8$ (range, 2 to 16). Distribution of various clinical presentations of the patients was shown in Table 2 .

The most common presentations of SLE patients were musculoskeletal and mucocutaneous manifestations. The vascular affection was as follows: venous affection including one patient with dural sinus thrombosis, one patient with femoropopliteal deep vein thrombosis (DVT), and four patients with infrapopliteal DVT and tibial arteries affection as peripheral arterial disease in four patients.

A total of 18 SLE patients (60\%) had positive anti-double stranded DNA antibody, eight patients (26.6\%) had positive anticardiolipin antibodies while 10 (33.3\%) had positive lupus anticoagulant antibodies. Lipid profile was higher in SLE patients compared to controls.

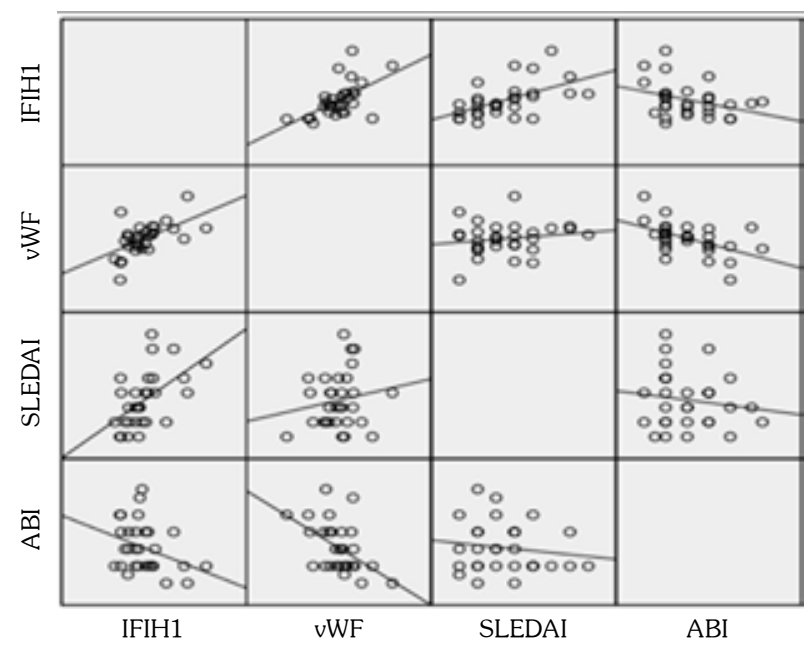

Figure 1. Correlation between IFIH1, vWF and different variables among the studied cases. IFIH1: Interferon-induced helicase C domain-containing protein 1; vWF: von Willebrand factor; SLEDAI: Systemic Lupus Erythematosus Disease Activity Index; ABI: Ankle Brachial Index. 
Table 5. Comparison between systemic lupus erythematosus patients with or without vascular manifestations

\begin{tabular}{|c|c|c|c|}
\hline \multirow[t]{2}{*}{ Variable } & $\begin{array}{l}\text { Vascular ( } \mathrm{n}=10,33.7 \%) \\
\quad \text { (arterial-venous) }\end{array}$ & No vascular $(n=20,66.7 \%)$ & \multirow[b]{2}{*}{$p$} \\
\hline & Mean \pm SD & Mean \pm SD & \\
\hline Cholesterol (mg/dL) & $206.6 \pm 74.9$ & $192.7 \pm 71.8$ & 0.403 \\
\hline High density lipoprotein (mg/dL) & $66.5 \pm 14.8$ & $47.6 \pm 15.6$ & 0.843 \\
\hline Low density lipoprotein (mg/dL) & $103.3 \pm 64.1$ & $99.7 \pm 40.6$ & 0.912 \\
\hline Triglycerides $(\mathrm{mg} / \mathrm{dL})$ & $149.9 \pm 60.9$ & $148.4 \pm 65.8$ & 0.692 \\
\hline Ankle Brachial Index & $0.8 \pm 0.1$ & $1.0 \pm 0.1$ & $0.008^{*}$ \\
\hline Carotid intima-media thickness (mm) & $6.6 \pm 1.4$ & $6.1 \pm 1.4$ & 0.195 \\
\hline Interferon-induced helicase $\mathrm{C}$ domain-containing protein 1 & $2.4 \pm 0.4$ & $1.3 \pm 0.3$ & $0.000 \neq$ \\
\hline Systemic Lupus Erythematosus Disease Activity Index & $10.3 \pm 2.3$ & $5.5 \pm 2.3$ & $0.000 \neq$ \\
\hline von Willebrand factor (IU/mL) & $7.1 \pm 1$ & $5.4 \pm 1.7$ & $0.000 \neq$ \\
\hline
\end{tabular}

A statistically significant difference was present between patients and controls as regards to IFHI1 gene expression, vWF, IMT, and ABI, which were higher in SLE patients compared to controls $(\mathrm{p}=0.0001)$ (Table 3).

A statistically significant positive correlation was present between IFIH1 gene expression and SLEDAI as well as vWF ( $r$-value 0.54 and 0.57 ; $p=0.002$ and $p=0.001$, respectively). However, ABI was inversely correlated with IFIH1 and vWF ( $\mathrm{r}$-value -0.34 and $-0.55 ; \mathrm{p}=0.05$ and $\mathrm{p}=0.02$, respectively). There was no statistically significant correlation between the other variables (Table 4, Figure 1).

In Table 5, the level of IFIH1 gene expression was compared between patients with arteriovenous (vascular) complications (33.7\%) and those without vascular manifestations (66.3\%). Results were higher in the former $(1.3 \pm 0.3$ vs. $2.4 \pm 0.4$, respectively) with a statistically significant difference $(p=0.000)$ and increased in patients with vascular manifestations with a mean of $2.3 \pm 0.4$ in venous and a mean of $2.5 \pm 0.4$ in arterial affection.

No statistically significant correlation was found between IFIH1 gene expression and the rest of the presenting manifestations of SLE including those with Raynaud's phenomenon $(p=0.65)$.

The concentration of $\mathrm{VwF}$ was statistically significantly higher in patients with vascular manifestations $(5.4 \pm 1.7)$ compared to those without $(7.1 \pm 1)(p=0.000)$. However, no significant difference was detected between patients with or without vascular manifestations regarding the lipid profile and carotid IMT.

There was no relationship between levels of IFIH1 gene with anti-double stranded DNA antibodyantibody, anticardiolipin antibodies or lupus anticoagulant antibodies $(p=0.92, p=0.06$, and $\mathrm{p}=0.47$, respectively).

Diagnostic performance of IFIH1 gene expression and vWF showed that they were valid inflammatory markers for SLE patients with vascular manifestations with the best cutoff values of 1.74 and 6.39, respectively (Figure 2, Table 6).

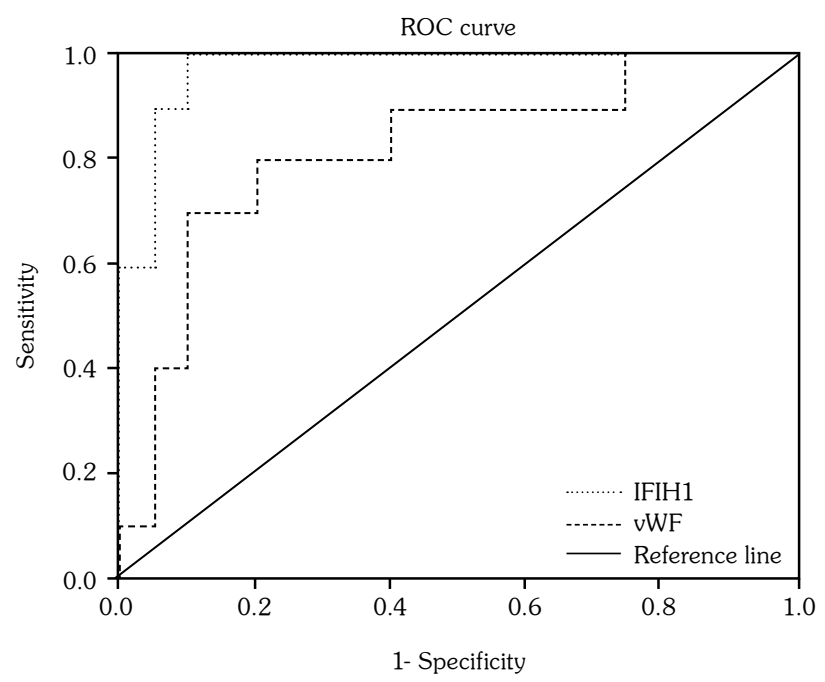

Figure 2. Roc curve of IFIH1 (SNP)rs 1990760 \& vWF in vascular affected systemic lupus erythematosus cases. IFIH1: Interferon-induced helicase C domain-containing protein 1; vWF: von Willebrand factor; ROC: Receiver Operating Characteristic. 


\begin{tabular}{|c|c|c|c|c|c|c|c|}
\hline \multirow[t]{2}{*}{ Variable } & \multirow[t]{2}{*}{ AUC } & \multirow[t]{2}{*}{$95 \% \mathrm{CI}$} & \multirow[t]{2}{*}{ Cutoff } & \multirow{2}{*}{$\frac{\text { Sensitivity }}{\%}$} & \multirow{2}{*}{$\frac{\text { Specificity }}{\%}$} & \multirow{2}{*}{$\frac{\mathrm{PPV}}{\%}$} & \multirow{2}{*}{$\frac{\mathrm{NPV}}{\%}$} \\
\hline & & & & & & & \\
\hline IFIH1 & 0.975 & $0.928-1.000$ & 1.74 & 100.0 & 90.0 & 83.3 & 100.0 \\
\hline vWF & 0.820 & $0.652-0.988$ & 6.39 & 80.0 & 80.0 & 66.7 & 88.9 \\
\hline
\end{tabular}

\section{DISCUSSION}

Systemic lupus erythematosus is an autoimmune disease, which affects almost all organs and tissues including skin, kidneys, lungs, musculoskeletal system, cardiovascular system, nervous system and serous membranes. ${ }^{10}$ Studies have reported the contribution of IFIH1 gene SNP in SLE susceptibility. ${ }^{6,11}$

The aim of our study was to evaluate IFIH1 gene expression by RT-PCR as a lupus associated gene and its role in the clinical manifestations of lupus, particularly vascular manifestations, comparing its level with other markers like ABI, and carotid IMT and vWF plasma level, which are surrogate markers of atherosclerosis and endothelial dysfunction.

Our results showed a statistically significant difference in IFIH1 gene expression between SLE patients and controls $(p=0.0001)$ with a mean of $1.7 \pm 0.6$ in SLE patients and a mean of $0.5 \pm 0.2$ in the control group. This comes in agreement with studies that included the Afro-Americans, Europeans ${ }^{4}$ and the Chinese population, ${ }^{12}$ demonstrating an association of the IFIH1 rs1990760 polymorphism with SLE susceptibility.

Disease activity was assessed using the SLEDAI, which ranged in our patients between 2 to 16 with a mean of $7 \pm 3$.8. Our results revealed a statistically significant positive correlation between IFIH1 and SLEDAI $(p=0.002)$. This conforms with the study of Petri et al. ${ }^{13}$ who found a positive correlation between other genes involved in the type 1 IFN pathway and SLEDAI score, denoting that IFIH1 gene is related to the disease activity through the transcription of type 1 IFN triggering other proinflammatory mediators in SLE.
When we compared the level of IFIH1 gene expression in patients with arteriovenous complications $(33.7 \%)$ to those without vascular manifestations (66.3\%), the results were higher in the former $(1.3 \pm 0.3$ vs. $2.4 \pm 0.4$, respectively) with a significant difference $(p=0.0001)$, standing for the association of IFIH1 over expression with vascular affection in SLE. This suggests the role of IFIH1 gene in apoptosis and inflammation ${ }^{4}$ resulting in endothelial dysfunction that has been symbolized as an early marker of subclinical atherosclerosis in SLE. ${ }^{14}$

However, we detected no significant correlation between IFIH1 gene expression and other presenting phenotypes including those with Raynaud's phenomenon similar to the results obtained by Cen et al., ${ }^{12}$ who showed no significant difference in IFIH1 gene expression among patients with or without individualized clinical features including musculoskeletal, mucocutaneous, neurologic, serositis and renal features; yet vascular manifestations were not investigated in that study.

We excluded patients with Raynaud's phenomenon from patients with vascular manifestations as affection of the digital capillaries is considered as a separate entity with different pathogenesis from those with larger vessels disease (proximal to the capillaries) in lupus. ${ }^{15}$

von Willebrand factor is a circulating glycoprotein that is synthesized in endothelial cells and released into the plasma in increased amounts by activated or damaged endothelial cells. Increased levels of vWf have been used for evaluation of endothelial dysfunction in SLE. ${ }^{8}$

Our study demonstrated a statistically significant difference in vWF levels between SLE patients and healthy controls $\mathrm{p}=0.0001$ similar to the studies of Habe et al. ${ }^{16}$ and Nienhuis et al., ${ }^{17}$ 
who found that levels of vWF were elevated in SLE patients compared to healthy controls $(p<0.001$ and $p \leq 0.05$, respectively). In addition, there was a significant difference between SLE patients and controls regarding $\mathrm{ABI}(\mathrm{p}=0.0001)$.

A meta-analysis study clearly showed that IMT was increased in SLE disease population compared with age- and sex-matched controls. ${ }^{18}$ This conforms to our study, which illustrated a statistically significant difference between SLE patients and controls in regards to IMT $(p=0.0001)$. This is also in concordance with findings of Khairy et al., ${ }^{19}$ who demonstrated a statistically significant difference between SLE patients and controls in terms of IMT $(p=0.001)$.

Furthermore, we assessed the variable markers in patients with vascular (arteriovenous) manifestations compared to those without vascular affection. We demonstrated that the concentration of vWF was statistically significantly higher in subjects positive for vascular affection $(5.4 \pm 1.7)$ relative to those who were negative $(7.1 \pm 1)(p=0.0001)$. A previous study had also compared patients with or without history of vascular affection and revealed a statistically significant difference $(p=0.001) .{ }^{20}$ Moreover, ABI in subjects with vascular affection was lower $(0.8 \pm 0.1)$ compared to those without vascular affection $(1.0 \pm 0.1)$, with a statistically significant difference $(p=0.008)$.

We detected no significant difference between patients with or without vascular affection regarding the lipid profile and CIMT ( $>>0.05)$. Furthermore, our study showed no significant difference between patients with arterial or venous affection regarding IFIH1 or vWF levels, indicating that venous affection was not benign compared to arterial disease as was previously thought. ${ }^{21}$

The strong positive relationship of IFIH1 and vWF with both types of vascular affection (arterial and venous) shows that both markers can indicate severe inflammation and ongoing destruction. IFIH1 gene may indicate endothelial dysfunction, increased plasma vWF levels and atherosclerosis in SLE. ${ }^{6,7}$ Our study results supported this hypothesis by showing a statistically significant positive correlation between IFIH1 and vWF as a marker of endothelial dysfunction $(p=0.001)$.
The finding of a significant negative correlation between IFIH1 gene and vWF with ABI $(p=0.05$ and $p=0.002$, respectively) is of great value, which confirmed the involvement of both IFIH1 gene and vWF in endothelial dysfunction in our SLE patients.

Concerning the relationship of endothelial dysfunction and IMT, it is known that atherosclerosis in patients with lupus has a long period of subclinical evolution. The first reversible step in the atherogenesis process is represented by endothelial dysfunction. Therefore, endothelial dysfunction occurs prior to the increase in IMT, which is a late step of atherosclerosis. ${ }^{17}$ This pertains to our results, which showed no correlation between IFIH1 gene expression and vWF (as markers of endothelial dysfunction) versus IMT ( $p=0.25$ and $p=0.33$, respectively), implying that some of our patients may have had endothelial dysfunction but did not develop overt atherosclerosis yet.

This may also be explained by the fact that increased IMT is a sign of diffuse atherosclerosis rather than localized atherosclerosis while vWF as a marker of inflammation and endothelial dysfunction may increase in case of any vascular activation and dysfunction whether localized or diffuse. ${ }^{22}$

However, our results did not show a statistically significant correlation between IFIH1 gene expression and vWF compared to traditional risk factors of atherosclerosis as age $(p=0.56$, $p=0.5$, respectively), disease duration $(p=0.6$, $p=0.34$, respectively) or lipid profile including total cholesterol ( $p=0.78, p=0.75$, respectively), LDL ( $p=0.50, p=0.20$, respectively) and triglycerides ( $p=0.35, p=0.45$, respectively).

This can be rationalized by the fact that patients with SLE have accelerated atherosclerosis even in young age. ${ }^{23}$ This could not be attributed to traditional Framingham risk factors such as altered lipid levels, aging and smoking alone, thus suggesting that autoimmunity contributes to accelerated atherosclerosis.

Moreover, Robinson et al. ${ }^{7}$ found that the IFIH1 rs1990760 risk allele $\mathrm{T}$ was associated with anti-DNA antibodies. This contradicted to our results, which showed an insignificant relationship between IFIH1 gene expression and immunological characteristics as regards to 
the presence of anti-DNA antibodies $(p=0.92)$, lupus anticoagulant $(p=0.47)$ and anticardiolipin antibodies $(p=0.06)$.

To our knowledge, this is the first study to evaluate IFIH1 gene in the Egyptian SLE patients and to correlate it to $\mathrm{vWF}$ and $\mathrm{ABI}$ to show its role in the vascular manifestations of the disease.

Our study has some limitations. Due to the small size of the study, we recommend further studies with larger scale to confirm the role of IFIH1 gene polymorphism in lupus susceptibility and its relationship to vascular manifestations and endothelial complications and also longitudinal prospective studies to evaluate IFIH1 gene expression as a reliable and reproducible predictor of premature atherosclerosis in high risk lupus patients. Also, we suggest further studies to be conducted to show any relationship between IFIH1 gene polymorphism and different SLE phenotypes.

In conclusion, IFIH1 gene expression was associated with Egyptian SLE patients and related to disease activity. IFIH1 gene expression was considered as a surrogate marker with superior sensitivity and validity than vWF with an emphasis on ongoing atherogenesis in Egyptian SLE patients with vascular affection. It may also serve as an early valid marker for endothelial dysfunction.

\section{Declaration of conflicting interests}

The authors declared no conflicts of interest with respect to the authorship and/or publication of this article.

\section{Funding}

The authors received no financial support for the research and/or authorship of this article.

\section{REFERENCES}

1. Perl A. Pathogenic mechanisms in systemic lupus erythematosus. Autoimmunity 2010;43:1-6.

2. Guerra SG, Vyse TJ, Cunninghame Graham DS. The genetics of lupus: a functional perspective. Arthritis Res Ther 2012;14:211.

3. Kahlenberg JM, Kaplan MJ. The interplay of inflammation and cardiovascular disease in systemic lupus erythematosus. Arthritis Res Ther 2011;13:203.
4. Molineros JE, Maiti AK, Sun C, Looger LL, Han $\mathrm{S}$, Kim-Howard $\mathrm{X}$, et al. Admixture mapping in lupus identifies multiple functional variants within IFIH1 associated with apoptosis, inflammation, and autoantibody production. PLoS Genet 2013;9:e1003222.

5. Winkler C, Krumsiek J, Lempainen J, Achenbach P, Grallert H, Giannopoulou E, et al. A strategy for combining minor genetic susceptibility genes to improve prediction of disease in type 1 diabetes. Genes Immun 2012;13:549-55.

6. Wang C, Ahlford A, Laxman N, Nordmark G, Eloranta ML, Gunnarsson I, et al. Contribution of IKBKE and IFIH1 gene variants to SLE susceptibility. Genes Immun 2013;14:217-22.

7. Robinson $\mathrm{T}$, Kariuki SN, Franek BS, Kumabe M, Kumar AA, Badaracco M, et al. Autoimmune disease risk variant of IFIH1 is associated with increased sensitivity to IFN- $\alpha$ and serologic autoimmunity in lupus patients. J Immunol 2011;187:1298-303.

8. Curiel RV, Bhagati R, Basavaraju L, Norton D, Katz $\mathrm{J}$, Haile $\mathrm{E}$, et al. Von Willebrand factor, red cell fragmentation, and disease activity in systemic lupus erythematosus. HSS J 2008;4:170-4.

9. Tyrrell PN, Beyene J, Feldman BM, McCrindle BW, Silverman ED, Bradley TJ. Rheumatic disease and carotid intima-media thickness: a systematic review and meta-analysis. Arterioscler Thromb Vasc Biol 2010;30:1014-26.

10. Liu Z, Davidson A. Taming lupus-a new understanding of pathogenesis is leading to clinical advances. Nat Med 2012;18:871-82.

11. Van Eyck L, De Somer L, Pombal D, Bornschein S, Frans G, Humblet-Baron S, et al. Brief Report: IFIH1 Mutation Causes Systemic Lupus Erythematosus With Selective IgA Deficiency. Arthritis Rheumatol 2015;67:1592-7.

12. Cen H, Leng RX, Wang W, Zhou M, Feng CC, Zhu Y, et al. Association study of IFIH1 rs1990760 polymorphism with systemic lupus erythematosus in a Chinese population. Inflammation 2013;36:444-8.

13. Petri M, Singh S, Tesfasyone H, Dedrick R, Fry K, Lal $\mathrm{P}$, et al. Longitudinal expression of type I interferon responsive genes in systemic lupus erythematosus. Lupus 2009;18:980-9.

14. Zardi EM, Afeltra A. Endothelial dysfunction and vascular stiffness in systemic lupus erythematosus: Are they early markers of subclinical atherosclerosis? Autoimmun Rev 2010;9:684-6.

15. Rosato E, Molinaro I, Pisarri S, Salsano F. Digital ulcers as an initial manifestation of systemic lupus erythematosus. Intern Med 2011;50:767-9.

16. Habe K, Wada H, Matsumoto T, Ohishi K, Ikejiri M, Tsuda K, et al. Plasma ADAMTS13, von Willebrand Factor (VWF), and VWF Propeptide Profiles in Patients With Connective Tissue Diseases and Antiphospholipid Syndrome. Clin Appl Thromb Hemost 2017;23:622-30. 
17. Nienhuis HL, de Leeuw K, Bijzet J, van Doormaal JJ, van Roon AM, Smit AJ, et al. Small artery elasticity is decreased in patients with systemic lupus erythematosus without increased intima media thickness. Arthritis Res Ther 2010;12:181.

18. Tyrrell PN, Beyene J, Feldman BM, McCrindle BW, Silverman ED, Bradley TJ. Rheumatic disease and carotid intima-media thickness: a systematic review and meta-analysis. Arterioscler Thromb Vasc Biol 2010;30:1014-26.

19. Khairy N, Ezzat $Y$, Naeem N, Taha R, Wesam R. Atherosclerosis biomarkers in female systemic lupus erythematosus patients with and without cardiovascular diseases. The Egyptian Rheumatologist 2017;39:7-12.
20. Gustafsson J, Gunnarsson I, Börjesson O, Pettersson S, Möller S, Fei GZ, et al. Predictors of the first cardiovascular event in patients with systemic lupus erythematosus - a prospective cohort study. Arthritis Res Ther 2009;11:186.

21. Pyrpasopoulou A, Chatzimichailidou S, Aslanidis S. Vascular disease in systemic lupus erythematosus. Autoimmune Dis 2012;2012:876456.

22. Lip GY, Blann A. von Willebrand factor: a marker of endothelial dysfunction in vascular disorders? Cardiovasc Res 1997;34:255-65.

23. Wilhelm AJ, Major AS. Accelerated atherosclerosis in SLE: mechanisms and prevention approaches. Int $\mathrm{J}$ Clin Rheumtol 2012;7:527-539. 